EPJ Web of Conferences 21, 04001 (2012)

DOI: $10.1051 /$ epjconf/20122104001

(C) Owned by the authors, published by EDP Sciences, 2012

\title{
Monte Carlo Simulation for Statistical Decay of Compound Nucleus
}

\author{
T. Kawano ${ }^{1, a}$, P. Talou, and M. B. Chadwick \\ Los Alamos National Laboratory, Los Alamos, NM 87545, U.S.A.
}

\begin{abstract}
We perform Monte Carlo simulations for neutron and $\gamma$-ray emissions from a compound nucleus based on the Hauser-Feshbach statistical theory. This Monte Carlo Hauser-Feshbach (MCHF) method calculation, which gives us correlated information between emitted particles and $\gamma$-rays. It will be a powerful tool in many applications, as nuclear reactions can be probed in a more microscopic way. We have been developing the MCHF code, CGM, which solves the Hauser-Feshbach theory with the Monte Carlo method. The code includes all the standard models that used in a standard HauserFeshbach code, namely the particle transmission generator, the level density module, interface to the discrete level database, and so on. CGM can emit multiple neutrons, as long as the excitation energy of the compound nucleus is larger than the neutron separation energy. The $\gamma$-ray competition is always included at each compound decay stage, and the angular momentum and parity are conserved. Some calculations for a fission fragment ${ }^{140} \mathrm{Xe}$ are shown as examples of the MCHF method, and the correlation between the neutron and $\gamma$-ray is discussed.
\end{abstract}

\section{Introduction}

To perform radiation transport calculations, the probabilities in nuclear process, such as neutron interaction or $\gamma$-ray production, are provided as input data to the simulation. This saves computational resources a lot. However, important correlations between neutrons and $\gamma$-rays cannot be taken into account exactly. A Monte Carlo simulation of the nuclear reaction process will be able to provide fully correlated information, i.e. all the neutrons and $\gamma$-rays emitted at an event obey the energy and angular momentum conservation laws. We have been developing a technique to solve the statistical HauserFeshbach theory [1] by means of a stochastic method, called Monte Carlo Hauser-Feshbach (MCHF) $[2,3]$. In our previous papers, the spin-dependence in the compound nucleus decay probabilities was summed before we perform the Monte Carlo simulations, which means the angular momenta are conserved in average only. This paper describes an extension of our MCHF calculations. A computer program, CGM (Cascading Gamma-rays and Multiplicity) [4] has been developed at Los Alamos, which can be combined with other transport simulation programs as an event-generator [5]. CGM runs in both stochastic and deterministic modes with very fine energy grid. This is particularly important to understand neutron emission at very low energies. In this paper we will also discuss the neutron evaporation process that can be studied with CGM.

\footnotetext{
a e-mail: kawano@lanl.gov

This is an Open Access article distributed under the terms of the Creative Commons Attribution-Noncommercial License 3.0, which permits unrestricted use, distribution, and reproduction in any noncommercial medium, provided the original work is properly cited.
} 


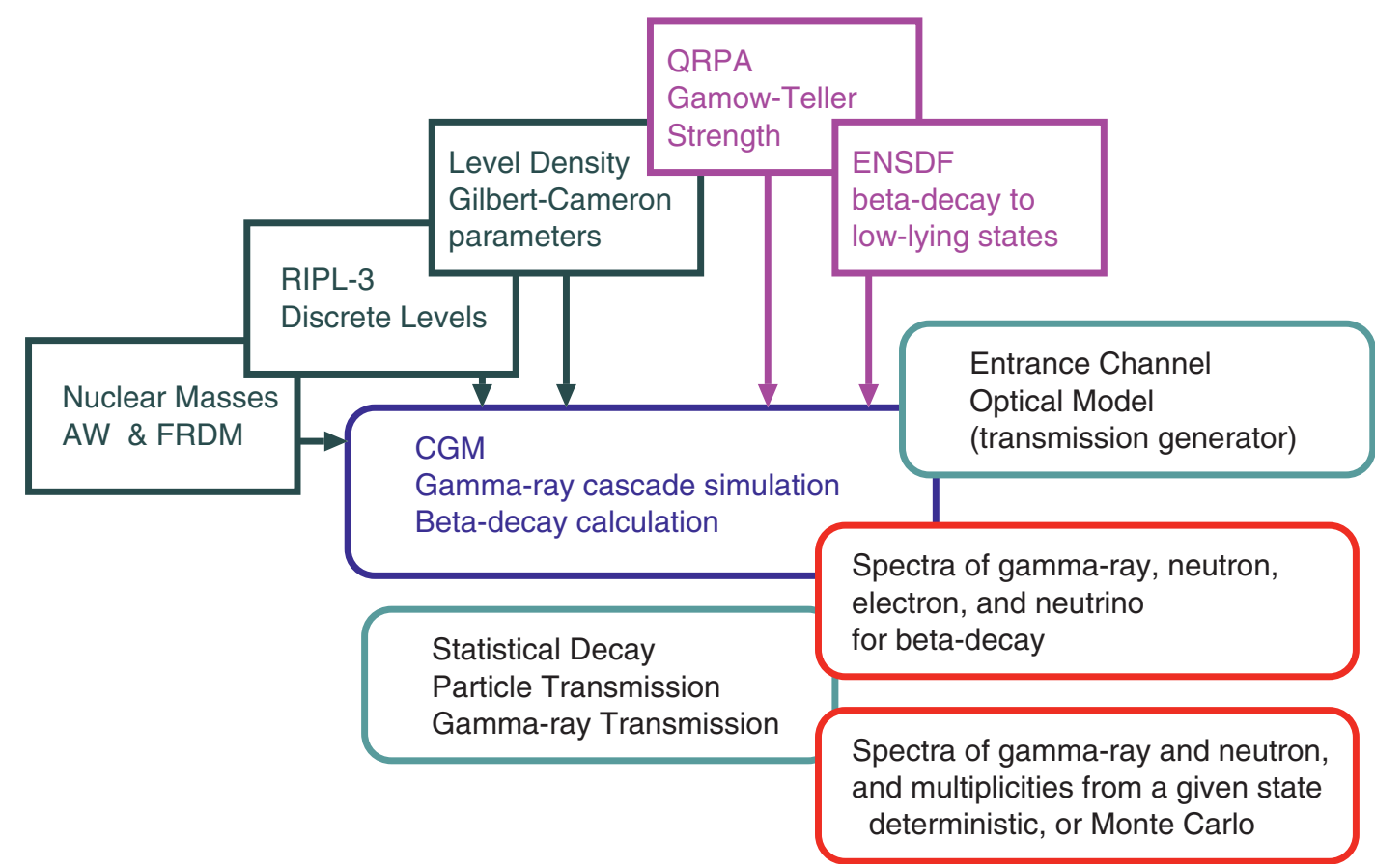

Fig. 1. Structure of the CGM code. The input quantities are shown by the boxes, and the output is shown by the two red rounded-boxes.

\section{CGM}

\subsection{Monte Carlo Method for Statistical Model}

The CGM code calculates the decay of a compound nucleus from a given excited state based on the Hauser-Feshbach formalism. CGM was developed mainly for three different purposes; (1) to study $\beta$-delayed neutron and $\gamma$-ray emission [6], (2) as an event generator in Monte Carlo radiation transport calculations, and (3) to study prompt fission neutron and $\gamma$-ray emissions [5]. The structure of this code is shown in Fig. 1.

In each application, an initial excitation energy $E_{x}$ of the compound nucleus $(Z, A)$ is provided, which may spread over different spin and parity states. In the Hauser-Feshbach theory, we calculate the transmission coefficients from all the possible final states to the compound state. The decay probabilities for $\gamma$-ray and neutron are given by

$$
P\left(\epsilon_{\gamma}\right) d E_{0}=\frac{T_{\gamma}\left(E_{x}-E_{0}\right) \rho\left(Z, A, E_{0}\right)}{N} d E_{0},
$$

for the $\gamma$-ray, where $\epsilon_{\gamma}=E_{x}-E_{0}$, and

$$
P\left(\epsilon_{n}\right) d E_{1}=\frac{T_{n}\left(E_{x}-S_{n}-E_{1}\right) \rho\left(Z, A-1, E_{1}\right)}{N} d E_{1}
$$

for the neutron, where $\epsilon_{n}=E_{x}-S_{n}-E_{1}, T_{n, \gamma}$ are the transmission coefficients, $\rho(Z, A, E)$ is the level density, $N$ is the normalization factor, $E_{0}$ and $E_{1}$ are the excitation energies in the residual nuclei, and $S_{n}$ is the neutron separation energy. The normalization factor is given by

$$
\begin{aligned}
N & =\int_{0}^{E_{x}} T_{\gamma}\left(E_{x}-E_{0}\right) \rho\left(Z, A, E_{0}\right) d E_{0} \\
& +\int_{0}^{E_{x}-S_{n}} T_{n}\left(E_{x}-S_{n}-E_{1}\right) \rho\left(Z, A-1, E_{1}\right) d E_{1} .
\end{aligned}
$$




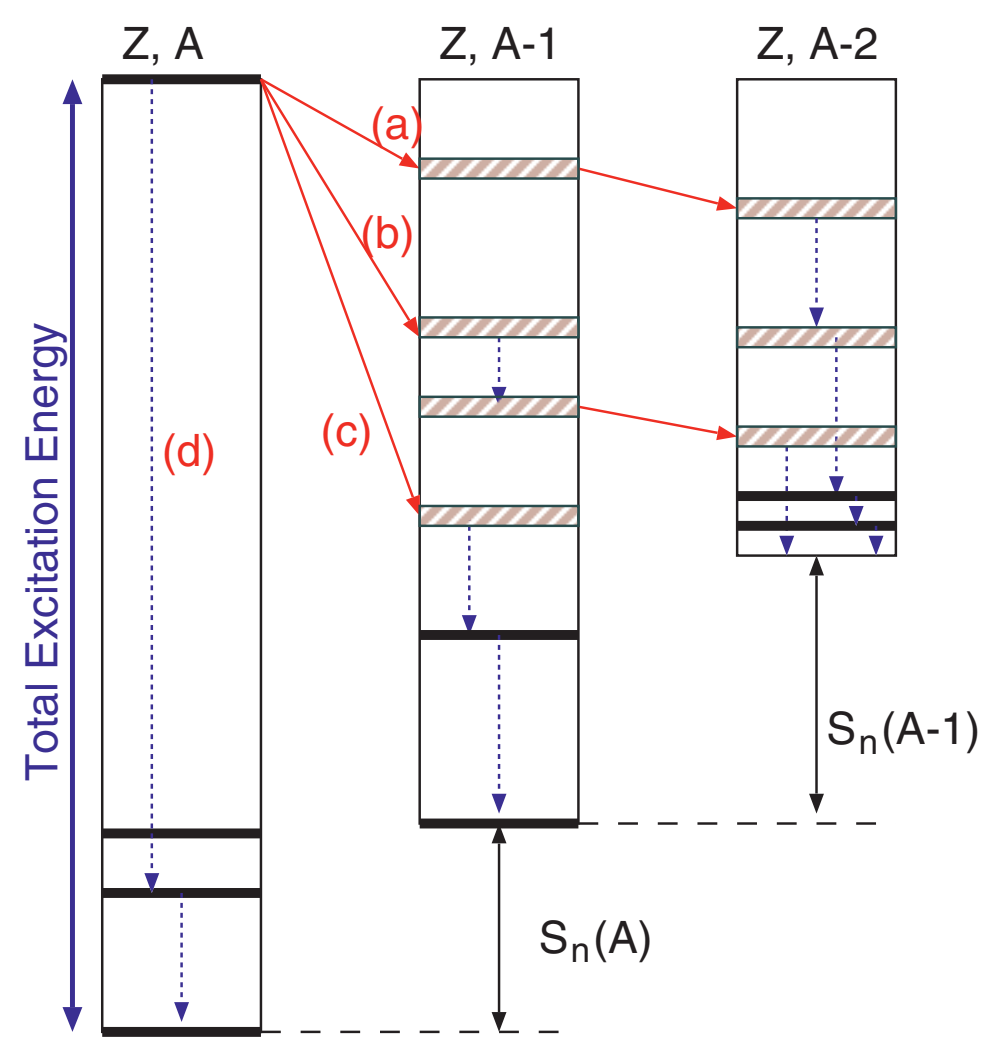

Fig. 2. Schematic picture of neutron and $\gamma$-ray emission calculation from a compound nucleus $(Z, A)$.

In the equations above we omitted indices of quantum numbers, but the integration is performed only for the spin and parity conserved states. At low excitation energies, the integration is replaced by a sum over the discrete levels.

The neutron transmission coefficients are obtained by solving the Schrödinger equation for optical model potentials. CGM has an built-in optical model solver with the Koning-Delaroche global optical potential [7]. The $\gamma$-ray transmission coefficient is calculated from the $\gamma$-ray strength function in a standard manner [8]. We include E1, M1, and E2 strength functions. A pygmy resonance and a scissors mode can be included if necessary. The level densities are calculated with the composite level density formulae of Gilbert and Cameron [9] with an updated parameterization [10].

First we calculate the decay probabilities $P\left(\epsilon_{n}\right)$ and $P\left(\epsilon_{\gamma}\right)$ from the initial state $\left(Z, A, E_{x}\right)$ (see Fig. 2) to the all possible final states, the next state $\left(\left(Z, A-1, E_{x}^{\prime}\right)\right.$, for example) is determined by a random sampling technique. We repeat this procedure until the nucleus reaches one of the discrete levels. At each stage the decay probabilities are calculated again to limit the amount of required memory. At the discrete levels, the $\gamma$-ray cascade is calculated in the Monte Carlo manner with the branching ratios given in the database.

\subsection{Variable Energy Bin Width}

In standard Hauser-Feshbach codes, such as GNASH [11], the continuum is discretized with a constant bin-width $\Delta E$. This makes computation faster and the algorithm simpler. However, information on neutrons whose energies are less than $\Delta E$ is inaccessible. In fact it was reported that an asymptotic form of low energy tail in the neutron emission spectra is different from the normal evaporation (or Weisskopf) form [12]. 
The Weisskopf spectrum,

$$
f(\epsilon) \propto \epsilon \exp \left(-\frac{\epsilon}{T}\right)
$$

has an asymptotic form of $\epsilon$ when $\epsilon \rightarrow 0$. The evaporation spectrum calculated with the HauserFeshbach theory consists of two quantities, the neutron transmission coefficients and the level densities. When the neutron energy is very low, the transmission coefficient is basically the $s$-wave component only,

$$
T_{0}=2 \pi S_{0} \propto \sqrt{\epsilon}
$$

where $S_{0}$ is the neutron strength function. We can assume that the level density is constant within a very narrow energy width, so that the Hauser-Feshbach evaporation spectrum has the asymptotic form of $\sqrt{\epsilon}$ at very low energies, which is the same as the Maxwellian shape, $\sqrt{\epsilon} \exp (-\epsilon / T)$. This was confirmed by the variable energy bin width calculations in CGM; the neutron emission spectra have the $\sqrt{\epsilon}$ shape up to $\sim 100 \mathrm{keV}$ for nuclei in the medium mass range. In the higher energy region, the Hauser-Feshbach spectra are well approximated by the Weisskopf shape.

\section{Results and Discussions}

\section{$3.1 \gamma$-ray Energy Spectra}

CGM provides a history of neutron and $\gamma$-ray emissions at each simulation. Starting from a neutron separation energy, where the neutron emission is suppressed, CGM generates a set of $\gamma$-ray cascades for the neutron capture process. This case is similar to the DICEBOX code [13].

We calculated the $\gamma$-ray cascade from ${ }^{239} \mathrm{U}$ at $4.81 \mathrm{MeV}$ excitation and $J^{\pi}=(1 / 2)^{+}$, which simulates experimental capture $\gamma$-rays for the thermal neutron induced capture reaction on ${ }^{238} \mathrm{U}$. The generated $\gamma$-rays were classified into different $\gamma$-ray multiplicities $m$, and the energy spectra for each $m$ were reconstructed. The top panel of Fig. 3 shows the color-coded histogram for the $\gamma$-ray spectra (production probabilities) for the multiplicity of $m=2 \sim 6$. For $m=1$, the spectrum becomes just a delta-function at $4.81 \mathrm{MeV}$.

In this calculation, we included $\gamma$-ray strength functions of E1, M1, and E2, as often adopted in nuclear reaction calculations. It is known that the two-step cascade $m=2$ is sensitive to the pygmy resonance or the scissors mode in the $\mathrm{MeV}$ region. In Fig. 3 lower panel shows the same calculation but a small M1 resonance $\left(E_{\gamma}=2 \mathrm{MeV}, \Gamma_{\gamma}=0.6 \mathrm{MeV}\right.$, and $\sigma_{0}=1.2 \mathrm{mb}$ [14] $)$ was added. The spectra for lower $m$ changed significantly, and the average $\gamma$-ray multiplicity became smaller by $5 \%$.

\subsection{Sequential Neutron Emission}

When the excitation energy of a compound nucleus is much higher than the separation energy, multiple neutron emission can occur. CGM calculates all the neutron and $\gamma$-ray competitions at each intermediate state until the excitation energy becomes less than the separation energy. In the current version of CGM, we are aiming at neutron-rich nuclei, such as fission fragments, so that the charged particle channels are ignored.

Figures 4,5 , and 6 show the calculated neutron and $\gamma$-ray energy spectra from excited ${ }^{140} \mathrm{Xe}$ at 10,15 , and $20 \mathrm{MeV}$. We performed $10^{5}$ simulations, which took about $1 \sim 2 \mathrm{~h}$ on a single CPU. The calculation time depends not only on the excitation energy but also the neutron separation energies and the out-gonig energy grid. As expected, the first neutron has the hardest spectrum, as the evaporation temperature becomes lower when more neutrons are emitted.

In this calculation we assumed that the initial spin distribution in the compound system is the same as that of the level density. When we calculate the neutron and $\gamma$-ray emission from fission fragments, the average spin $\langle J\rangle$ could be much higher than our assumption, and careful assessment of the initial spin distribution is needed. 

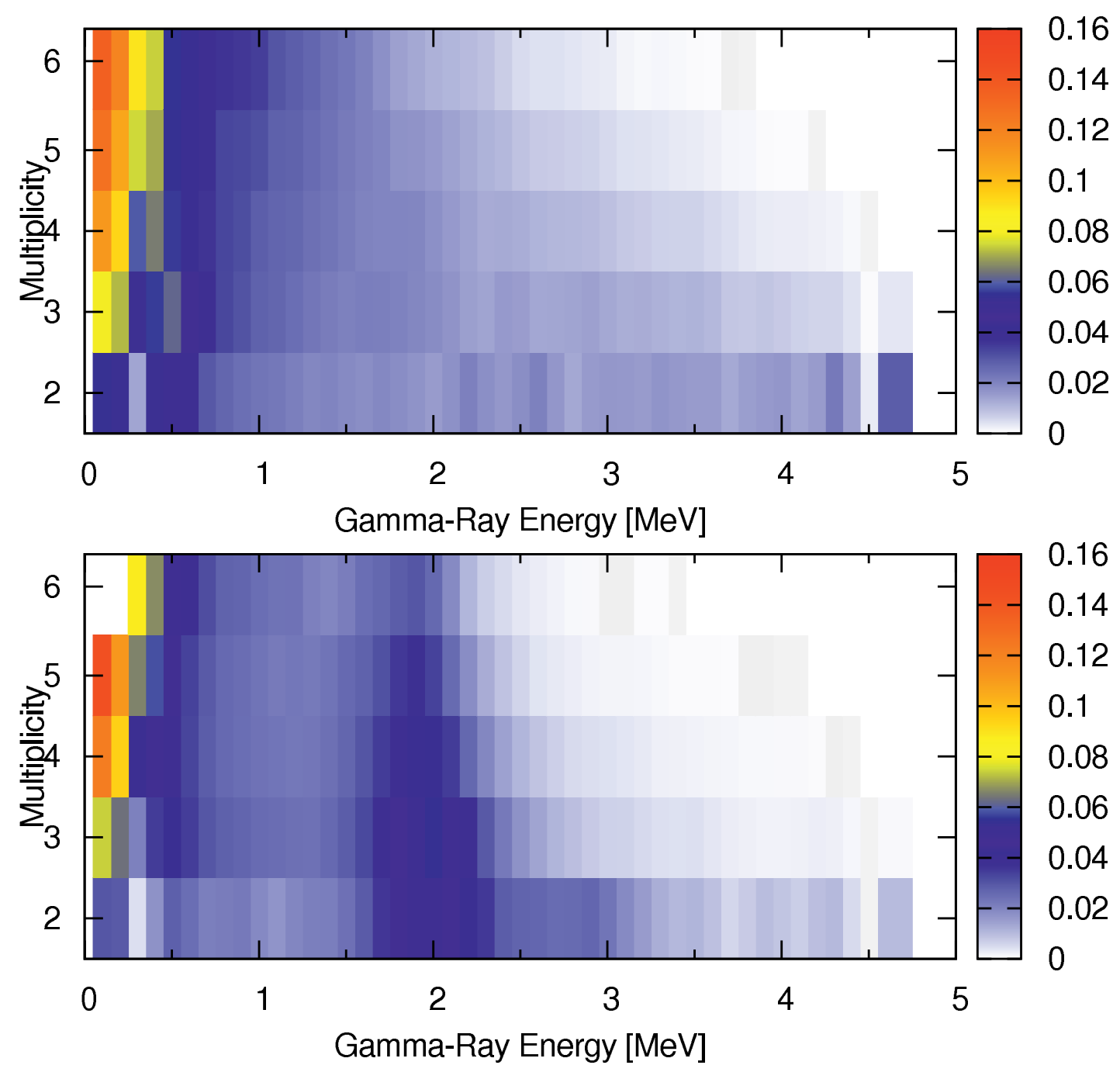

Fig. 3. The $\gamma$-ray energy spectra from the thermal neutron capture reaction on ${ }^{238} \mathrm{U}$ for different $\gamma$-ray multiplicities. The top panel calculation includes general E1, M1, and E2 strength functions, and the lower panel is the case when the scissors mode is added.

\subsection{Neutron and $\gamma$-ray Correlations}

The MCHF technique allows us to study relationship between the emitted neutrons and $\gamma$-rays. These correlations are often the result of energy and angular momentum conversations. They sometimes show a nuclear structure effect too. In Fig. 7 the calculations are the same as in Figs. $4-6$ but the results are presented in a different way — the neutron energy spectra for individual $\gamma$-ray multiplicity. The $\gamma$-ray multiplicity distribution is also shown on the right column. It does not look the $\gamma$-ray multiplicity distribution is a simple function of nuclear excitation. When the initial excitation energy is just above the sum of all neutron separation energies, the last neutron tends to leave the residual nucleus in its low-lying state, or even in its ground state, hence the average $\gamma$-ray multiplicity becomes smaller.

These multiplicity distributions change when the initial spin distribution becomes different, as noted before. As an example, we calculated the same neutron spectra and the $\gamma$-ray multiplicity distributions as in Fig. 7 but for the different spin distribution case. The spin cut-off parameter $\sigma^{2}$ in the 
EPJ Web of Conferences

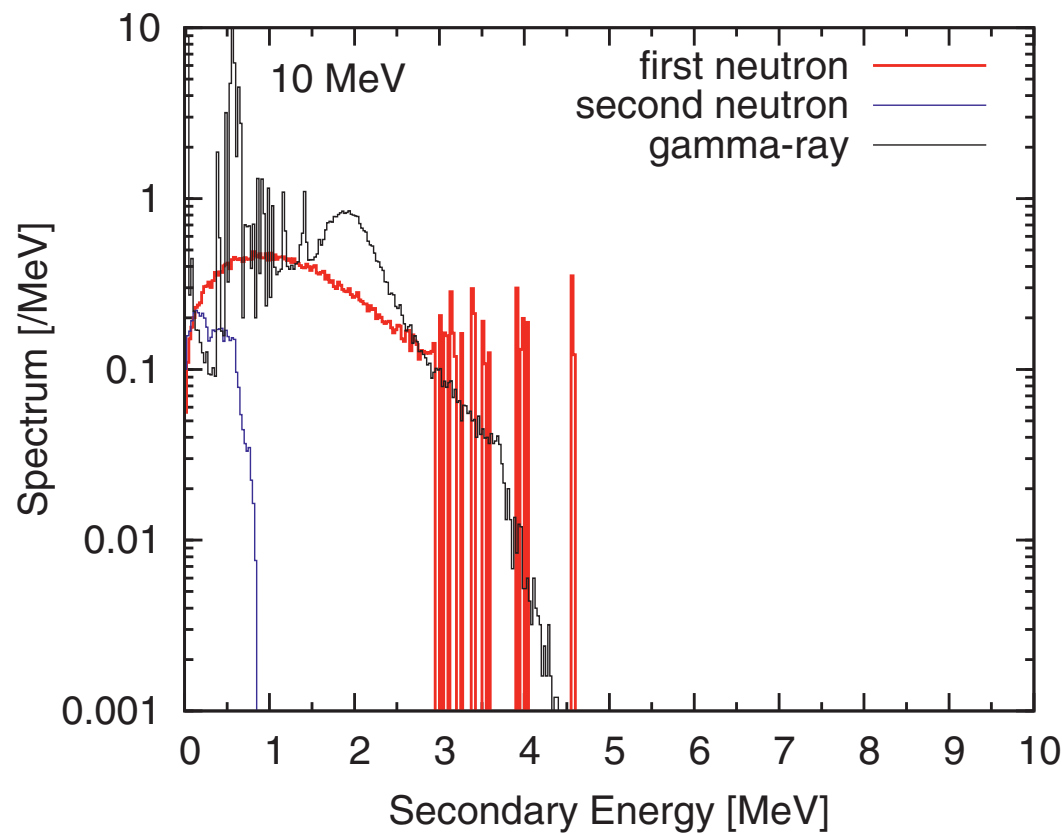

Fig. 4. The Monte Carlo simulated neutron $\gamma$-ray energy spectra from ${ }^{140} \mathrm{Xe}^{*}$ at $10 \mathrm{MeV}$ excitation energy. The red, blue, and green histograms are for the first, second, and third emission of neutrons, and the black histogram is for the total $\gamma$-ray.

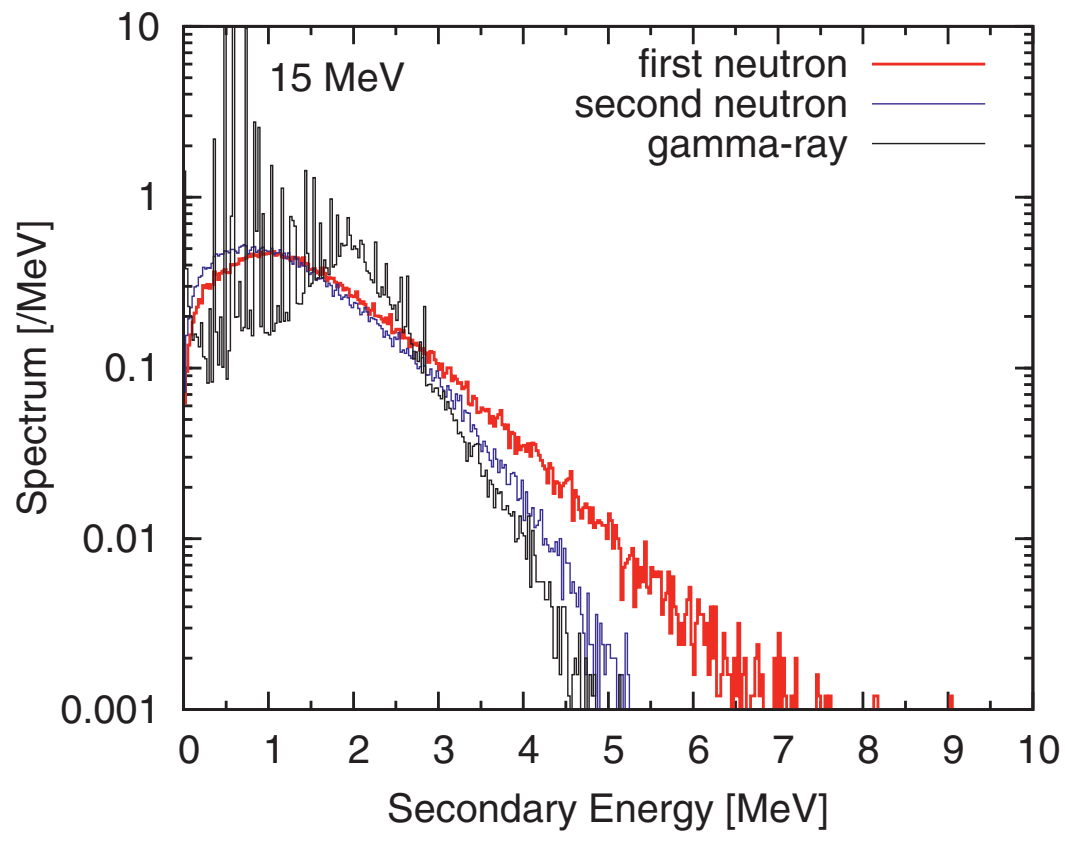

Fig. 5. Same as Fig. 4 but for $15 \mathrm{MeV}$. 


\section{$\mathrm{CNR} * 11$}

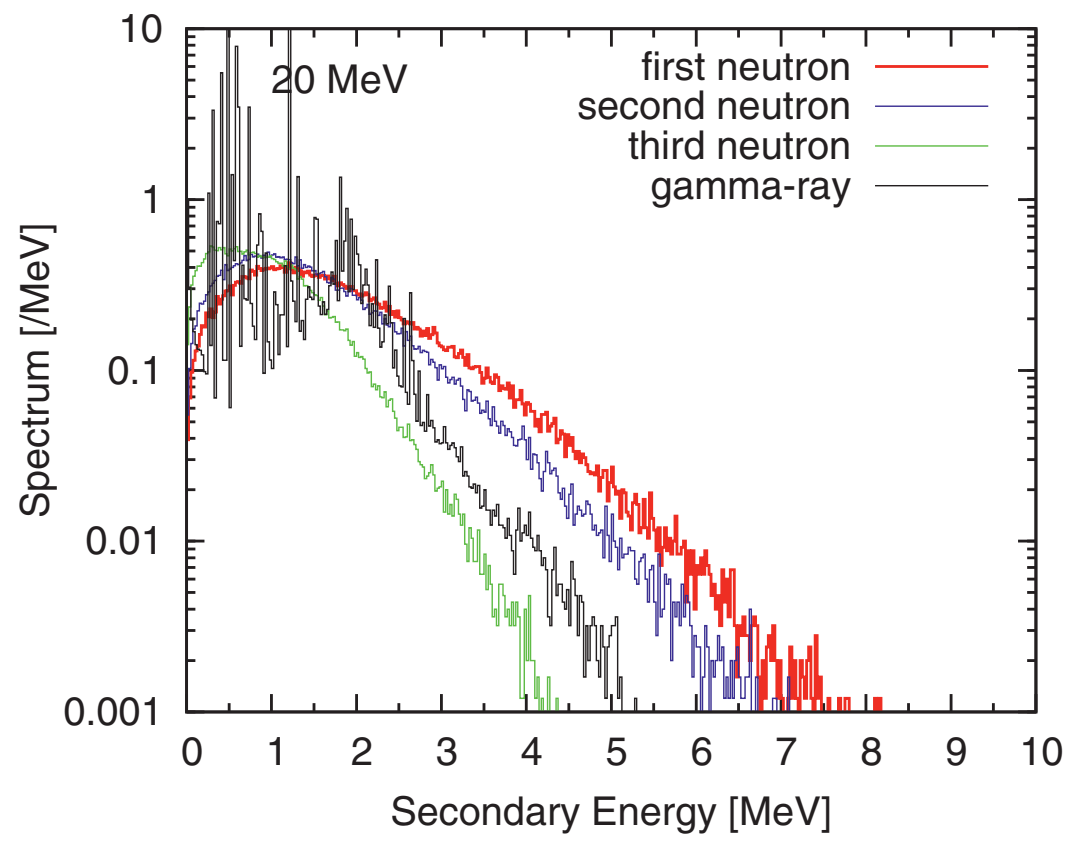

Fig. 6. Same as Fig. 4 but for $20 \mathrm{MeV}$.

Gaussian-shape spin distribution,

$$
R(J)=\frac{2 J+1}{2 \sigma^{2}} \exp \left\{-\frac{(J+1 / 2)^{2}}{2 \sigma^{2}}\right\},
$$

was doubled, and the results are shown in Fig. 8. The average $\gamma$-ray multiplicity becomes the difference in $J$ between the compound and ground states becomes larger, while the E1 transition changes the spin by only $1 \hbar$. This also impacts on the neutron emission from the compound system. When MCHF is combined with experimental data on prompt $\gamma$-ray emission, we can obtain more information on nuclear reaction mechanisms that cannot be easily seen in traditional nuclear reaction experiments or simulations.

\subsection{Conclusion}

The CGM code developed at LANL can be applied to many different compound nucleus decay processes to study properties of neutron and $\gamma$-ray emissions. In this paper we introduced our Monte Carlo Hauser-Feshbach (MCHF) method, and the Monte Carlo simulations for the neutron and $\gamma$-ray emissions from a compound nucleus were performed. As examples, calculated results of compound nucleus decay from excited ${ }^{140} \mathrm{Xe}$ were shown. The results include not only the energy spectra of neutrons and $\gamma$-rays, as can also be obtained from the traditional deterministic method, but also the neutron energy spectra for a specific $\gamma$-ray multiplicity $m$. These quantities are more sensitive to the initial spin distribution in the compound system, and therefore will provide better insights into the nuclear reaction mechanism. 


\section{EPJ Web of Conferences}
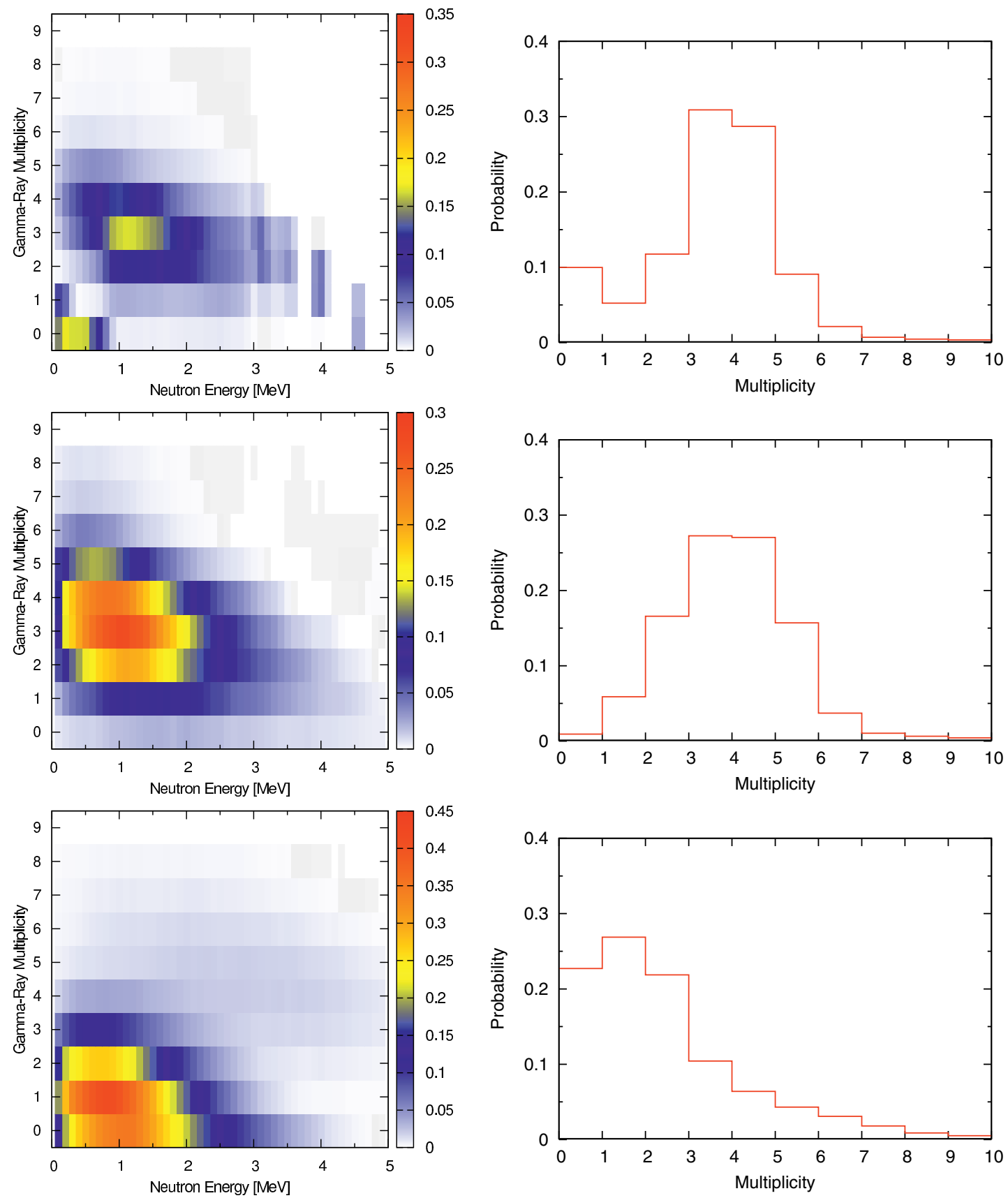

Fig. 7. The neutron energy spectra (left) and the $\gamma$-ray multiplicity distributions (right) from ${ }^{140} \mathrm{Xe}^{*}$ at $10 \mathrm{MeV}$ (top panel), $15 \mathrm{MeV}$ (middle), and $20 \mathrm{MeV}$ (bottom) excitation energies for each $\gamma$-ray multiplicity. 
CNR*11
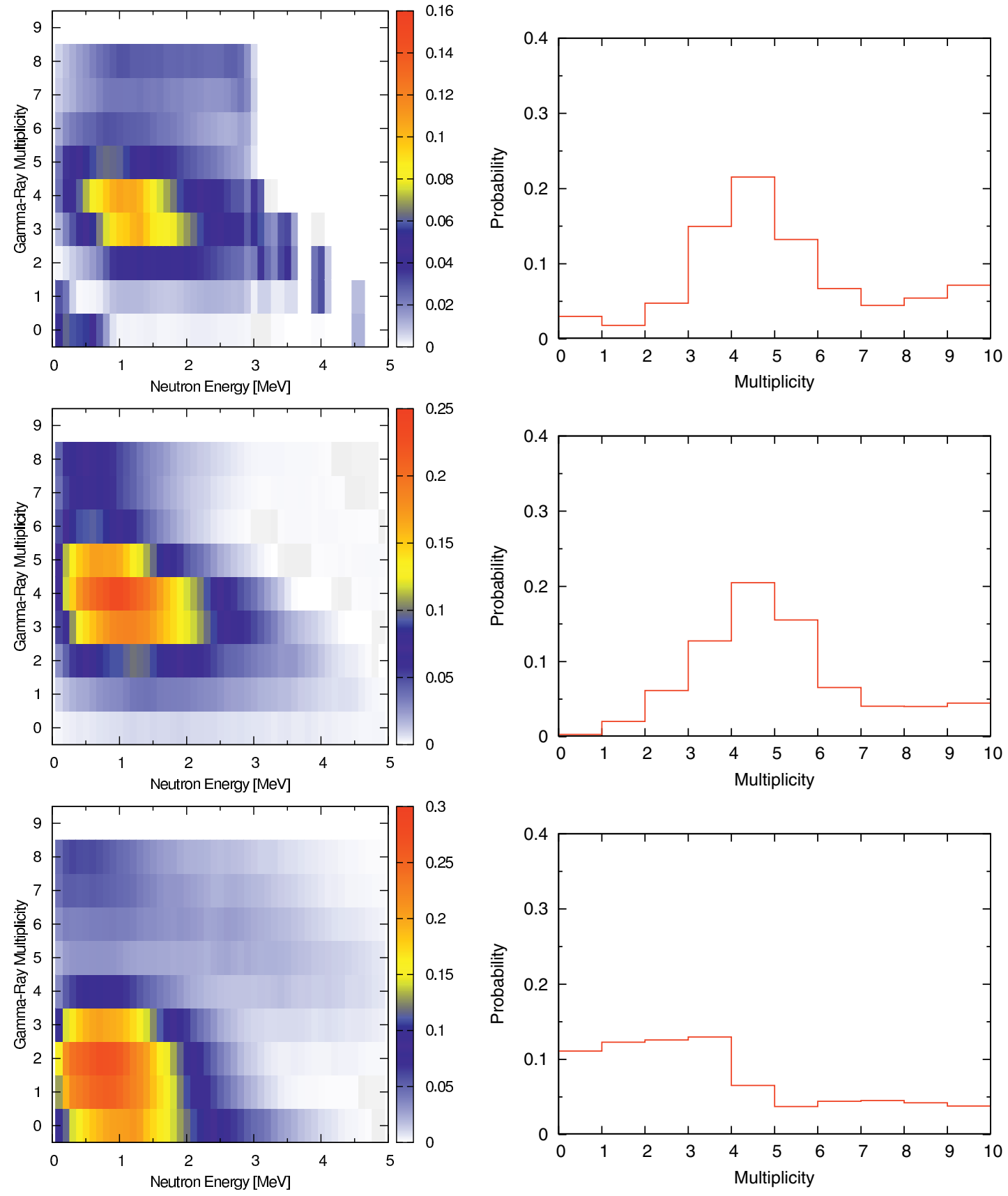

Fig. 8. Same as Fig. 7, but the initial spin distribution is changed. 
EPJ Web of Conferences

\section{Acknowledgment}

We would like to thank M. Krtička of Charles University, Prague, and J. Ullmann of LANL for valuable discussions. This work was carried out under the auspices of the National Nuclear Security Administration of the U.S. Department of Energy at Los Alamos National Laboratory under Contract No. DE-AC52-06NA25396.

\section{References}

1. W. Hauser, H. Feshbach, Phys. Rev. 87, 366 (1952)

2. T. Kawano, P. Talou, M. B. Chadwick, T. Watanabe, J. Nucl. Sci. Technol., 47, 462 (2010)

3. T. Kawano, P. Talou, M. B. Chadwick, S. Holloway, P. Möller, T. Watanabe, J. Korean Phys. Soc. 59, 785 (2011)

4. T. Kawano, S. T. Holloway, CGM, Cascading Gamma-Ray and Multiplicity, ver.3, LACC-11-018 Los Alamos National Laboratory (2011)

5. P. Talou, B. Becker, T. Kawano, M. B. Chadwick, Y. Danon, Phys. Rev. C 83, 064612 (2011)

6. S. Holloway, T. Kawano, P. Möller, J. Korean Phys. Soc. 59, 875 (2011)

7. A. Koning, J. P. Delaroche, Nucl. Phys., A713, 231 (2003)

8. J. Kopecky, M. Uhl, Phys. Rev. C, 41, 1941 (1990)

9. A. Gilbert, A. G. W. Cameron, Can. J. Phys., 43, 1446 (1965)

10. T. Kawano, S. Chiba, H. Koura, J. Nucl. Sci. Technol., 43, 1 (2006); T. Kawano, "updated parameters based on RIPL-3," (unpublished, 2009)

11. P. G. Young, E. D. Arthur, M. B. Chadwick, "Comprehensive nuclear model calculations: Theory and use of the GNASH code," Proc. of the IAEA Workshop on Nuclear Reaction Data and Nuclear Reactors - Physics, Design, and Safety, Singapore: World Scientific Publishing, Ltd., for Trieste, Italy, April 15 - May 17, 1996. Eds. A. Gandini and G. Reffo, pp. 227-404 (1996)

12. R. E. MacFarlane, (private communication, 2010)

13. F. Becvar, Nucl. Instrum. Meth. Phys. Res. A417, 434 (1998)

14. M. Krtička, (private communication, 2011) 